\title{
Verification of the Esbjerg-Nørskov relation from small-cluster calculations
}

\author{
A. N. Andriotis \\ Theoretical and Physical Chemistry Institute, National Hellenic Research Foundation, \\ 48 Vasileos Constantinou Avenue, GR-116 35 Athens, Greece
}

(Received 13 May 1987)

\begin{abstract}
The use of small-cluster calculations in testing the applicability of the Esbjerg and Nørskov's equation, $E_{\text {rep }}(\mathbf{r})=\alpha_{\text {eff }} \rho(\mathbf{r})$, which relates the repulsive interaction $E_{\text {rep }}(\mathbf{r})$, between a helium atom and a metal surface with the unperturbed electron density $\rho(\mathbf{r})$ of the probed metal, is bound to inherent limitations. As is shown in the example case of the $\mathrm{H}-\mathrm{H}-\mathrm{He}$ trimer, $\alpha_{\text {eff }}$ depends on the geometry of this trimer. On the other hand, in the case of helium dimers, it is shown that $\alpha_{\text {eff }}$ depends on the internuclear distance and on the energy of the valence electron of the host atom.
\end{abstract}

Many recent investigations related to the diffraction of thermal helium atoms from solid surfaces have concentrated their emphasis on the applicability of the Esbjerg and Nørskov's equation, ${ }^{1}$ i.e.,

$$
E_{\text {rep }}(\mathbf{r})=\alpha_{\text {eff }} \rho(\mathbf{r}) \text {, }
$$

which relates the unperturbed electron density $\rho(\mathbf{r})$ of the metal probed by helium atoms with the repulsive heliummetal interaction, $E_{\text {rep }}(\mathbf{r})$. Of particular interest are the results of the cluster calculations of Bagus, Nelin, Batra, and Bauschlicher, ${ }^{2}$ who studied the interaction between a helium atom and the $\mathrm{O} / \mathrm{Ni}(001)$ surface. In particular, they found that when the helium atom probes the surface region along a line perpendicular to the surface and in the middle of two oxygen atoms (center position), $\alpha_{\text {eff }}$ is much larger than when the helium atom probes the same surface region along a line perpendicular to the surface and passes through the center of an oxygen atom (on top position). They attributed this difference to the fact that the surface charge-density contours are strongly corrugated with respect to the size of the $\mathrm{He}$ atom. Although such an explanation can account for most of the calculated difference in the values of $\alpha_{\mathrm{eff}}$, we would like to note that reasons inherent to small-cluster calculations can be partly responsible for such observed limitations of Eq. (1).

One such inherent reason may become apparent by noting that the greatest contribution to $E_{\text {rep }}(\mathbf{r})$ is due to the process of orthogonalization of the helium orbitals to those of the substrate (cluster). As the orthogonalization procedure depends on the geometry and the size of the cluster, one can expect different values of $\alpha_{\text {eff }}$ for various geometries of the combined He-substrate cluster.

We have tested these arguments in the case of smallcluster calculations. In particular, we performed Hartree-Fock (HF) calculations for two geometries of the helium trimer H-H-He. However, before we proceed with the trimer calculations, we will report some results on the helium dimers. There are two specific reasons for which such a test study is very useful.

(i) For hydrogen-helium dimers and/or trimers there exist very accurate calculations, which include correlation effects to a high degree of approximation. ${ }^{3-5}$ The existence of such calculations enables us to find out whether correlation effects affect the general form of Eq. (1) and if so, to what extent. It is noted that such questions dominate in the theories, which either include the correlation effects of the helium-metal interaction within the localdensity approximation (LDA), or they separate the repulsive contribution of the helium-metal interaction from the correlation van der $\mathrm{Waals}^{6}$ or the hybridization one. ${ }^{7}$

(ii) Our recent theoretical investigations ${ }^{8-10}$ showed that in the case of the interaction of a helium atom with a metal surface, $\alpha_{\text {eff }}$ can be expressed in terms of the characteristics of both the metal and the helium atom. Among the former characteristics, we mention the singleelectron effective potential of the metal and its work function. These observations indicate a dependence of $\alpha_{\text {eff }}$ on the helium-metal distance and the energy of the valence electrons of the metal probed. Such a dependence is expected to be more pronounced in the case of the helium dimers.

In all our calculations we used the POLYATOM program, ${ }^{11}$ which ensures for fully self-consistent results at a nonlocal HF level of approximation. For the $\mathrm{He}$ atom, Huzinaga's ${ }^{12} 7 s$ set of Gaussian-type orbitals (GTO's) was used uncontracted. For the hydrogen atom, we used an uncontracted $7 s$ GTO basis set, namely, Huzinaga's ${ }^{12}$ $6 s$ set as enlarged by adding one diffuse $s$ function according to Römelt, Peyerimhoff, and Buenker. ${ }^{4}$ Finally, for the $\mathrm{Li}$ atom, we used the uncontracted $7 s$ GTO set of Roos and Siegbahn. ${ }^{13}$

In the case of helium dimers, $E_{\text {rep }}$ takes the form

$$
E_{\text {rep }}(R)=E_{\mathrm{HeX}}(R)-E_{\mathrm{He}}^{\mathrm{free}}-E_{X}^{\mathrm{free}},
$$

where $R$ is the internuclear distance of the dimer $\mathrm{He} X$ and $E_{X}^{\text {free }}$ is the HF energy of the free atom $X=\mathrm{H}, \mathrm{He}$, or Li. Then, $E_{\text {rep }}(R)$ is plotted against the electron density $\rho_{X}(R)$ of the unperturbed $X$ atom, i.e.,

$$
\rho_{X}(R)=\sum_{i} n_{i}\left|\psi_{X}^{(i)}(R)\right|^{2},
$$

where the summation runs over the occupied orbitals whose occupancies are $n_{i}$. For the cases of the three dimers studied, it is observed that for interaction energies smaller than 100-200 meV (depending on the dimer) Eq. (1) is applicable. At this energy region, on the average, we found $\alpha_{\mathrm{eff}}^{(\mathrm{H})}=855, \alpha_{\mathrm{eff}}^{(\mathrm{He})}=2438, \alpha_{\mathrm{eff}}^{(\mathrm{Li})}=351 \mathrm{eV} a_{0}^{3}$ from the slope of the corresponding graphs. The superscript as- 
TABLE I. Values of the ratio $E_{\text {rep }} / \rho$ as a function of the internuclear distance of the dimer $\mathrm{He} X, X=\mathrm{H}, \mathrm{He}, \mathrm{Li}$.

\begin{tabular}{cccc}
\hline \hline $\begin{array}{c}R \\
\text { (a.u.) }\end{array}$ & $\mathrm{H}$ & $\begin{array}{c}E_{\text {rep }}(R) / \rho(R)\left(\mathrm{eV} a_{0}^{3}\right) \\
\mathrm{He}\end{array}$ & $\mathrm{Li}$ \\
\hline 2.50 & 200 & 1143 & 402 \\
3.00 & 237 & 1634 & 291 \\
3.50 & 425 & 2174 & 275 \\
4.00 & 758 & 2639 & 288 \\
4.50 & 859 & 3619 & 309 \\
5.00 & 917 & 4323 & 331 \\
5.50 & & & 351 \\
6.00 & & & 368 \\
7.00 & & & 394 \\
\hline \hline
\end{tabular}

signed to $\alpha_{\text {eff }}$ specifies the dimer $\mathrm{He} X$ to which it corresponds. At higher interaction energies the values $\alpha_{\mathrm{eff}}^{(X)}$ become smaller. In the case of $\mathrm{HeH}$, we compared our Hartree-Fock [self-consistent field (SCF)] results with those which include correlation effects at a high level of approximation $^{3}$ [although using $\mathrm{HF}$ results for $\rho_{x}(R)$ ]. Once again we find that for interaction energies smaller than $100 \mathrm{meV} \mathrm{Eq.} \mathrm{(1)} \mathrm{is} \mathrm{applicable.} \mathrm{However,} \mathrm{the} \mathrm{es-}$ timated value of $\alpha_{\mathrm{eff}}$ is $30 \%$ smaller than that found with the $\mathrm{HF}$ energy results.

In Table I we indicate the dependence of the HF approximated ratio $E_{\text {rep }}(R) / \rho(R)$ on the internuclear distance $R$ for the three dimers studied. It is observed that $\alpha_{\text {eff }}$ exhibits a rather strong dependence on $R$. One common feature, which becomes apparent from these results, is that at large internuclear distances [small values of $\left.\rho_{X}(R)\right] \alpha_{\text {eff }}$ increases with the distance. However, in the case of the He-Li dimer an increase in $\alpha_{\text {eff }}$ is observed also at small internuclear distances. On the other hand, if we plot the average values of $\alpha_{\mathrm{eff}}^{(X)}, X=\mathrm{H}, \mathrm{He}, \mathrm{Li}$, of the weak interaction region against the orbital energy $I_{X}$ of the outer electron orbital of the free $X$ atom, it is found that to a good approximation

$$
\alpha_{\mathrm{eff}}^{(X)}=\beta I_{X}^{k}
$$

where $\beta=38.5$ and $k=1.30\left(\alpha_{\mathrm{eff}}^{(X)}\right.$ in eV $a_{0}^{3}$ and $I_{X}$ in eV).

In the case of the $\mathrm{H}-\mathrm{H}-\mathrm{He}$ trimer we used a $\mathrm{H}-\mathrm{H}$ internuclear distance, $R=3.0$ a.u. Two geometries of the trimer $\mathrm{H}-\mathrm{H}-\mathrm{He}$ were checked. The $A$ geometry, where the
TABLE II. Distances $R_{A}$ and $R_{B}$ (in a.u.) of the helium atom from the $\mathrm{H}-\mathrm{H}$ axis and the corresponding repulsive interactions $E_{\text {rep }}^{(A)}$ and $E_{\text {rep }}^{(B)}$ (in $\mathrm{meV}$ ) for the two geometries $A$ and $B$ (see text) of the trimer $\mathrm{H}-\mathrm{H}-\mathrm{He}$

\begin{tabular}{|c|c|c|c|c|}
\hline$R_{A}$ & $R_{B}$ & $E_{\text {rep }}^{(A)}$ & $(\mathrm{meV})$ & $E_{\mathrm{rep}}^{(B)}$ \\
\hline 3.50 & 3.39 & 415 & & 363 \\
\hline 4.00 & 3.88 & 191 & & 173 \\
\hline
\end{tabular}

He nucleus is located along the line perpendicular to the middle of the interhydrogen distance $R$. The $B$ geometry assumes that the helium atom is located at the line perpendicular to $R$ and passing through one hydrogen nucleus. We assign the symbols $R_{A}$ and $R_{B}$ to the distances of the helium atom from the interhydrogen line in the geometries $A$ and $B$, respectively. Our test is limited at two pairs of positions $\left(R_{A}, R_{B}\right)$, where the unperturbed electron density of the $\mathrm{H}-\mathrm{H}$ dimer is the same. For each trimer geometry specified by $R_{A}$ and $R_{B}$ we found $E_{\text {rep }}^{(i)}$, $i=A, B$ by simply generalizing Eq. (2). Our results, which are included in Table II, imply that the values of

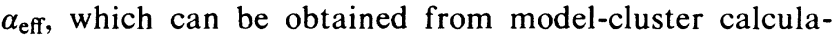
tions, depend on the geometry of the cluster considered. Although, in the present case, the difference found is about $10 \%$, other cases might exist where this difference can be greater. This will depend on the kind of molecular orbitals involved and on the way these orbitals change as we change the geometry of the cluster. Thus, the present results indicate that, in addition to the observed ${ }^{10}$ inclinations of the Esbjerg and Nørskov relation when applied to jellium-type surfaces, small-cluster calculations used to verify this relation are bound to inherent limitations. It is not clear yet if this limitation reflects a more general one, which is associated with the degree or the absence of delocalization of the electron states probed by a helium atom. On the other hand, this may account for part of the difference in the values of $\alpha_{\text {eff }}$ found by Bagus et al. ${ }^{2}$ who considered two positions of the helium atom above the $\mathrm{O} / \mathrm{Ni}(001)$ surface.

I would like to thank Dr. G. Theodorakopoulos for useful discussions and for providing me with numerical results prior to publication.
${ }^{1}$ N. Esbjerg and J. K. Nørskov, Phys. Rev. Lett. 45, 807 (1980).

2P. S. Bagus, C. J. Nelin, I. P. Batra, and C. W. Bauschlicher, Jr., Phys. Rev. B 35, 441 (1987).

${ }^{3}$ G. Theodorakopoulos, I. D. Petsalakis, C. A. Nicolaides, and R. J. Buenker, J. Phys. B (to be published).

${ }^{4}$ J. Römelt, S. D. Peyerimhoff, and R. J. Buenker, Chem. Phys. 34, 403 (1978).

${ }^{5}$ S. C. Farantos, Mol. Phys. 54, 835 (1985).

${ }^{6}$ E. Zaremba and W. Kohn, Phys. Rev. B 15, 1769 (1977).
${ }^{7}$ J. F. Annett and R. Haydock, Phys. Rev. B 34, 6860 (1986).

${ }^{8}$ A. N. Andriotis, Phys. Rev. B 33, 1482 (1986).

${ }^{9}$ A. N. Andriotis, Solid State Commun. 59, 761 (1986).

${ }^{10}$ A. N. Andriotis and C. A. Nicolaides, Phys. Rev. B 35, 2583 (1987).

${ }^{11}$ Hunt's GVBONE version.

${ }^{12}$ S. Huzinaga, J. Chem. Phys. 42, 1293 (1965).

${ }^{13}$ B. Roos and P. Siegbahn, Theor. Chim. Acta (Berlin) 17, 209 (1970). 